\title{
XXVII. The mixing of gases.-Remarks on Mr. Burbury's recent papers
}

Prof. W.McF. Orr M.A.

To cite this article: Prof. W.McF. Orr M.A. (1908) XXVII. The mixing of gases.-Remarks on Mr. Burbury's recent papers, Philosophical Magazine Series 6, 15:86, 297-300, DOI: $10.1080 / 14786440809463770$

To link to this article: http://dx.doi.org/10.1080/14786440809463770

曲 Published online: 16 Apr 2009.

Submit your article to this journal ¿

Џll Article views: 3

Q View related articles $₫$ 
XXVII. The Mixing of Gases,-Remarks on Mr. Burbury's recent Papers. By Prof. W. McF. Oni, M.A.*

T BEG to make some remarks on the two papers by 1 Mr. Burbury on the Diffusion of Gases which have recently appeared in this Magazine $t$.

Mr. Burbury appears to dispute the propositions: (1) that when two gases mix by diffusion the process is an irreversible one ; (2) that every irreversible process is attended by a loss of available energy.

As regards the former proposition, Mr. Burbury, in the first paper, after discussing some points in the kinetic theory of gases, suggests $\$$ that the diffusion would be reversible, provided the system were completely isolated, a condition which it is impossible to realize in nature, but admits that it is irreversible in any experiments that can practically bo made. On this view, the point at issue may be said by one unfamiliar with the kinetic theory of gases to be an abstract question affecting a system under conditions so unnatural that, whichever way it is decided, it nevertheless allows the natural process to be, as a matter of fact, irreversible.

Mr. Burbury does not discuss the latter of the two propositions directly, but turns his attention instead to an equivalent theorem relating to increase of entropy, and merely for the particular case in hand, $i . e$, the diffusion of gases. On this point he urges $\S$ that there is no gain of entropy in any one collision between molecules, and that therefore no increase can result from all the collisions taken together. It may, I think, be fairly replied, again from a standpoint outside the kinetic theory, that it is quite unnecessary to consider such questions as this and others which he raises. The usual definition of entropy makes no reference to single molecules but deals with matter in bulk; the Kinetic Theory of Gases is an interesting annexe of Thermodynamics, but the latter in no way depends on the former ; if the molecular theory gives results which contradict our experience, necessarily limited to that of gross matter, then, as happens from time to time with other branches of science, it requires revision, as regards logical development, or premisses, or both. Moreover, a being who could have experience of individual molecules would, in all probability, not understand the notions of pressure, temperature, heat,

* Communicated by the Author.

$\uparrow$ Phil. Mag. July and Sept. 1907.

\pm L. c. p. 125.

L. c. p. 124. 
work, energy, \&c., in the sense in which we adopt them; our distinction between heat and other forms of kinetic energy might seem to him specially artificial, and in this connexion it may be remarked that, although it is impossible to separate a mixtare of (perfect) gases into constituents at the same pressure as the mixture, if the temperature is kept constant, without doing work on them, yet the gases give out in the process an equivalent quantity of heat, so that their energy remains unaltered.

The second proposition in all its generality, or the equivalent theorem concerning entropy, does not, indeed, appear to be formally deducible from any usually accepted statement of Thermodynamic laws; each may form his own opinion as to how far experience and experiment warrant its acceptance. I imagine, however, that it is admitted almost universally that every process occurring in vature is irreversible and also that it involves a loss of available energy.

But in the particular case of the diffusion of gases, or at least of some gases, it is a fact established by direct experiment, and, I had supposed, beyond all dispute whatever, that there is a loss of available energy, a statement which Mr. Burbury appears to challenge * (for the case of oxygen and nitrogen). Lord Rayleigh, in the opening paragraph of the paper to which $\mathrm{Mr}$. Burbury refers in his second paper, called attention to this in 1875 ; and the rest of Lord Rayleigh's paper is devoted, not so much to a proof that there is such a loss, but to calculations of its amount. In the simple and common experiment there described, a tube containing hydrogen and closed at the upper end stands over water; the hydrogen escapes through the pores more rapidly than the air enters, thus creating a partial vacuum, and the water accordingly rises. The available energy is thus actually seen to be used, to some extent, in pumping water up a glass tube. And, if we were able to separate the hydrogen and the atmospheric air, in their original states, from the mixture, keeping the temperature constant, without doing work on the system-a process the possibility of which Mr. Burbury appears to suggest in his second paper $\uparrow$-it would be easy to devise a heat engine by which we could continue to pump water from a lower to a higher level, and in which the working substance would go through a closed cycle taking in heat at a constant temperature (atmospheric), but requiring no condenser,-thus obtaining a perpetual

* L. c. p. 123.

† L. c. p. 423 . 
motion of the second kind, as Ostwald and Planck call it; it seems unnecessary to describe a suitable arrangement in detail. There would, of course, be a loophole of escape if there were any reason to think that the energy is actually drawn from the plug.

Mr. Burbury makes no allusion to this paragraph of Lord Rayleigh's, but proceeds to refer to one of the possible processes of separation of gases which Lord Rayleigh employed in calculating the amount of work necessary for the operation. In this it is supposed that a tall narrow vertical tube is mounted on a large reservoir containing a mixture of two gases at sensibly constant pressure: the composition varies in different parts of the tube, owing to the effect of gravity, there being a greater percentage of the lighter gas at the top of the tube than at the bottom. Lord Rayleigh supposes that a small quantity of gas is (1) removed from the top of the tube (an equivalent amount being, I take it, automatically supplied from the reservoir so that the composition of the gas at every point of the tube remains unaltered); (2) compressed until it attains the pressure of the gas in the reservoir ; (3) allowed to fall to the level of the reservoir; and (4) forced into the reservoir, (but, as I understand it, kept separated from the gas in the reservoir by an impermeable membrane); the temperature is supposed to be constant throughout. Thus, from the original contents of the reservoir there has been separated a small portion of gas which is of a different composition from that which remains. Mr. Burbury, however, apparently ascribes to Lord Rayleigh a somewhat different process; he supposes each element of the vertical column, in succession, brought down to the level of the reservoir (but without being replaced by other similar elements and without being forced into the reservoir). This he describes as a partial separation of the gases, which appears to be a misdescription; it is merely an alteration in the position and density of gases without any change in the proportions of their constituents. He then regards these operations as the second half of a complete cycle of which he proceeds to supply the first half : evidently he does not use the phrase "complete cycle" in its usual sense. In this first half he supposes a tube, which is a facsimile of the vertical one, but placed horizontally at the level of the reservoir, to be filled with the reservoir gases, interchanges the molecules of the two gases in it until each element has the same constitution as the corresponding element of the vertical column, expands each element until it has the same pressure and density as the 
corresponding element of the vertical column, and sets the tube vertical in a position corresponding to that of the column. When these operations are prefixed to those which he ascribes to Lord Rayleigh, a series of processes is obtained which, of course, might as well be terminated after the first, $i . e$. , the partial separation of the gases in the horizontal tube; and Mr. Burbury contends that Lord Rayleigh has proved nothing inconsistent with the supposition that this separation -at constant pressure and constant temperature-can be performed without the expenditure of work. In one sense this contention may be admitted, for, if I apprehend the situation correctly, Lord Rayleigh's investigation is not directly relevant to the series of processes which Mr. Burbury describes. If, however, in the reservoir, a small portion of gas of the same constitution as that at the top of Lord Rayleigh's tube be separated from the remaining contents, and if this partial separation can be performed at constant temperature and constant pressure without doing any work on the gases, then, by reversing the processes which Lord Rayleigh, if I understand correctly, actually does employ, it follows from his investigation that the gas system would again constitute the working substance in a heat engine which contradicts the Second Law of Thermodynamics.

\section{Intelligence and Miscellaneous Articles.}

ON THE FOCOMETRY OF A CONCAVE LENS.

To the Editors of the Philosophical Magazine.

Gentlemen,-

TT has just been pointed out to me that the first method I described in the "Note on the Focometry of a Concave Lens" in the Philosophicnl Magazine for January, is given in the Syllabus of the Course of Practical Instruction in Physics at the Royal College of Science, London (1902).

I was, unfortunately, unaware of this at the time of writing, as it is not given in my copy, which is an earlier edition (1892). The second method is, of conrse, similar in principle to the first; while the third is derived from the second by reversing the direction of the rays.

Yours faithfully, J. A. Tomoriss. 